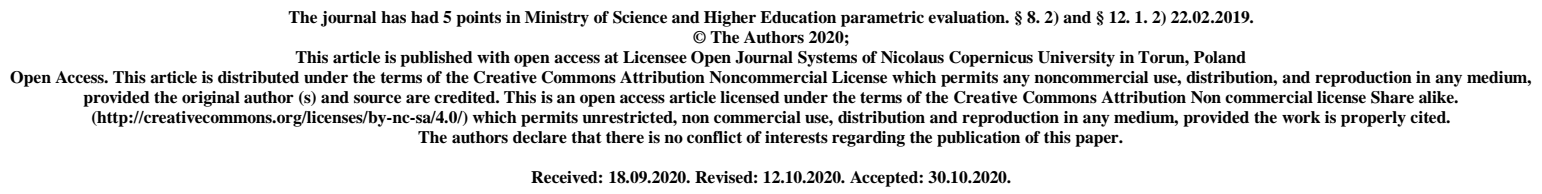

\title{
ABACTERIAL PROSTATITIS OF VIRAL ETIOLOGY AND ITS CHRONOBIOLOGICAL FEATURES
}

\author{
V. S. Goydyk, M. V. Novikov, A. I. Gozhenko
}

\section{State Enterprise "Ukrainian Research Institute of Transport Medicine, Ministry of Health of Ukraine", Odessa}

\begin{abstract}
Urogenital infections are a global health burden all over the world. The combination of general "aging" of the population and improvement of diagnostic capabilities led to a surge in the detection of diseases of the urogenital sphere, much of which have viral etiology. An increase in the load on doctors of the urological profile, especially working at outpatient units, dictates the need to improve the efficiency of their work. The use of mathematical modeling methods in the study of medical records of 600 men with urogenital diseases made it possible to ascertain the seasonality of requests for medical help, as well as to create a prognostic model, the use of which suggests the possible number of referrals for patients with abacterial prostatitis of viral etiology during any month of the next year.
\end{abstract}

Key words: urogenital infections; mathematical modeling; Gaussian distribution; second degree polynomial.

Today the problem of formalization of urological medical tasks remains urgent. Its value is especially growing in terms of application of the principles and methods of evidencebased medicine not only for timely identification of the patient's state but also, accordingly, for reliable and accurate diagnosis in urological practice. There are two important trends in modern medicine. Due to life prolongation, the mass share of diseases incident to elderly, 
increases. At the same time, there is an increase in the proportion of these diseases detected due to improved diagnostic capabilities at a younger age [1]. These trends also include such a significant field as urology. Diseases of the genitourinary system are becoming more common, and given their impact on the patients' quality of life, create new challenges for doctors of all levels. Therefore, the new data that will accelerate the diagnostic and treatment process are very relevant.

Among infectious diseases of the genitourinary system, an important role in the prevalence and frequency of visits to the doctor is played by lesions of its lower parts, the socalled urogenital infections (UGI). The range of UGI pathogens is extremely wide, including bacteria, fungi, protozoa, viruses [2, 3]. However, it is viral lesions that come to the fore, including due to the spread of modern research methods. They can be both an independent etiological factor and a trigger mechanism for the development of diseases caused by opportunistic flora [4]. As a result, primarily the most vulnerable parts of the genitourinary system suffer, and the most common forms of urogenital diseases, urethrites and prostatites, proceeds [5]. Of all the variety of viruses known as the etiological factor in lesions of the genitourinary system, herpesviruses take up aan important place [6, 7].

In general, herpesvirus lesions are attracting increasing attention of the scientific community. Their role in the development of diseases of many organs and systems has been proved. The variety of manifestations of the disease, the tendency to long-term and constant recurrence determine the need to advance our knowledge about Herpesviridae family and this concerns representatives of almost all medical specialties $[8,9,10]$.

In the general structure of urological diseases, chronic prostatitis $(\mathrm{CP})$, according to various authors, lies in 2-3rd place by prevalence rate. Among adult men aged 20-50 years, it affects almost half, and in men over 50 years, about $30 \%$ of diseases are CP or its combination with benign or malignant prostate tumors [11, 12]. At the same time, the constant recurrence of the disease, microsymptoms, which persist for a long time, significantly impair the quality of life, up to the development of psychosomatic disorders, neuroses and depression $[13,14]$.

Given the complexity of UGI etiology, as well as the extraordinary diversity of pathogenesis, the greater use of various statistical methods that are effectively employed to correctly and accurately describe mass phenomena $[15,16]$ are necessary for further study and formalization of the data.

Along with improvement of diagnosis and treatment of urological diseases, the general problem of assessing and predicting the incidence of UGI more clearly outlines the systemic 
task of rapid, reliable and accurate diagnosis, which requires not only special medical but also systemic adaptation [17].

The specificity of such tasks is in the interrelation of many conditional and unconditional factors, which complicates their formalization and finding an adequate model of diagnostic examination, and also significantly affects the results of the formation of control effects on the patient in the form of appropriate action [18].

In order to quantify the impact and identify qualitatively significant etiological factors of urological diseases manifestations, we conducted an expert survey among urologists. As a result, one of the most informative factors of significant influence, which received a high concordance coefficient in this group of experts (equal to 0.85), a chronobiological factor, namely, the seasonality of the disease was called.

To study, establish and quantify the most significant factors influencing the manifestations of UGI of herpesvirus etiology, we previously conducted a survey that showed the importance of seasonality. This is a trend in the dynamics of which a pronounced and certain frequency of urological diseases [19] exacerbation is clearly visible. Despite some publications available in the literature, this topic, in our opinion, is still insufficiently studied in relation to urology.

The objective: to study the chronobiological features (seasonal recurrence) of manifestations and exacerbations of viral etiology prostatites. All the patients under examination visited an outpatient urologist.

Materials and methods. The study was conducted during $2011-2015$ and 2017 on the basis of the polyclinic № 20 (Odessa, Ukraine) and contained the reports of an outpatient urologist. The results of examination and treatment of 600 patients have been analyzed. Given the presented data on comorbidities and the results of the examination, some of the initial number of patients were excluded.

Exclusion criteria:

- the presence of urogenital pathology with dominant manifestations of another etiological factor;

- severe somatic diseases;

- hepatocellular insufficiency;

- significant deviations of laboratory parameters: transaminases, creatinine levels, electrolyte imbalance, etc.;

- hypersensitivity to any drug. 
Thus, 178 male patients were selected. They were diagnosed with prostatitis of viral etiology (herpesvirus, cytomegalovirus or a combination thereof). The group under examination consisted of 150 persons aged $18-65$ y. o. (mean $33.0 \pm 2.7$ ). They completed the outpatient course of treatment $(2011$ - 2015) in elective order. The predominance of a certain social stratum was not revealed. The control group of patients was comparable to the main one by age ( $p>0.05)$ included 28 persons (2017).

All patients underwent a general clinical and laboratory examination: collection of complaints and history, physical data, general analysis of blood and urine, determination of serum biochemical parameters (levels of total protein, bilirubin, creatinine, urea, glucose), tests to confirm herpesvirus etiology of the disease ( EIA, PCR), ultrasound of the abdominal cavity and genitourinary system.

The data from fully completed in accordance with the existing guidelines forms were evaluated.

The full data set had a minimum number of missed records and was $80-100 \%$ of its content.

This circumstance allows us to consider the surveyed sample representative and suitable for further statistical analysis. Determination of criteria and basic calculations were performed using a statistical package demo version of the program Statistica-12 [1, 2, 13, 14].

Results and discussion. The distribution of the patients by age is shown in Table 1 . The evidence presented suggests that the largest group of the patients (almost a half) aged 20 29 y.o. This is the most mobile and socially active population group. The next most frequent age group (30 - 39 y.o.), constituted almost a quarter of the patients under examination.

Table 1

Distribution of the patients by age, $n=150$

\begin{tabular}{|c|c|c|}
\hline Age & Number of persons & $\%$ \\
\hline Up to 20 & 8 & 5,3 \\
\hline $20-29$ & 69 & 46,0 \\
\hline $30-39$ & 32 & 21,3 \\
\hline $40-49$ & 24 & 16,0 \\
\hline $50-59$ & 10 & 6,7 \\
\hline$>60$ & 7 & 4,7 \\
\hline Всього & 150 & 100 \\
\hline
\end{tabular}

Quite a high percentage of patients in the older age group (50 - 65 years) can be explained by seeking help due to recurrence of long-standing, insufficiently treated diseases. 
Table 2 shows the duration of the disease until one seeks for medical help separately for each age group. According to the anamnesis, these values vary from 0.5 to $10-12$ years.

Table 2

The average duration of the disease (years) in different age groups

\begin{tabular}{|c|c|c|}
\hline Age group, years & Minimum value & Maximum value \\
\hline Up to 20 & 0,5 & 1,5 \\
\hline $20-29$ & 0,5 & 3 \\
\hline $30-39$ & 1,5 & 5 \\
\hline $40-49$ & 1,5 & 7 \\
\hline $50-59$ & 2 & $10-12$ \\
\hline$>60$ & 2 & $9-12$ \\
\hline
\end{tabular}

When the age increases age, the maximum duration of the disease increases $(p<0.05)$, too. This is due to the predominant infection with herpesviruses at a young age, the long course of the disease, accompanied by relapses. Patients with a disease duration of 1.5 years or more indicate that they have previously been examined and treated, but without a positive result. Recurrences of the disease, which are not accompanied by clear clinical manifestations, in many cases were not taken into account by patients, which was also one of the reasons of its chronicity.

We have obtained a significant amount of measurements under almost identical conditions and they are independent of each other, but are characterized by the influence of many factors of relatively low intensity, then, according to the law of large numbers, they have Gaussian or close to it, distribution. Therefore, the next step in the study of sampling parameters was to check the normality of the distribution of the number of patients (Fig. 1).

Verification of the sample by the Shapiro-Wilk test showed that the value of the criterion is $0.9, \mathrm{p}=0.21>0.05$, i. e. the null hypothesis $\mathrm{H} 0$ is true (the distribution of the obtained values does not contradict the Gaussian).

Since the distribution of patients by quantitative (age) is not exactly Gaussian, but is sufficiently approximated by it, it is necessary to apply standard methods of mathematical statistics to further describe the observed trends with some caution. However, there is evidence that a deviation of $20-25 \%$ is not fatal, which corresponds to our results (deviation within 10\%). Thus, we can, without making a methodological error, use the methods of parametric statistics.

In general, if we do not take into account the first group of patients aged 18 - 20 y.o. and taking into account the normality test, there is a steady age trend of decreasing the 
number of patients, which according to experimental data using standard statistical programs can be approximated by exponent $\mathrm{Y}=$ Aeex $(-\mathrm{kt})$, where $t$ is the time.

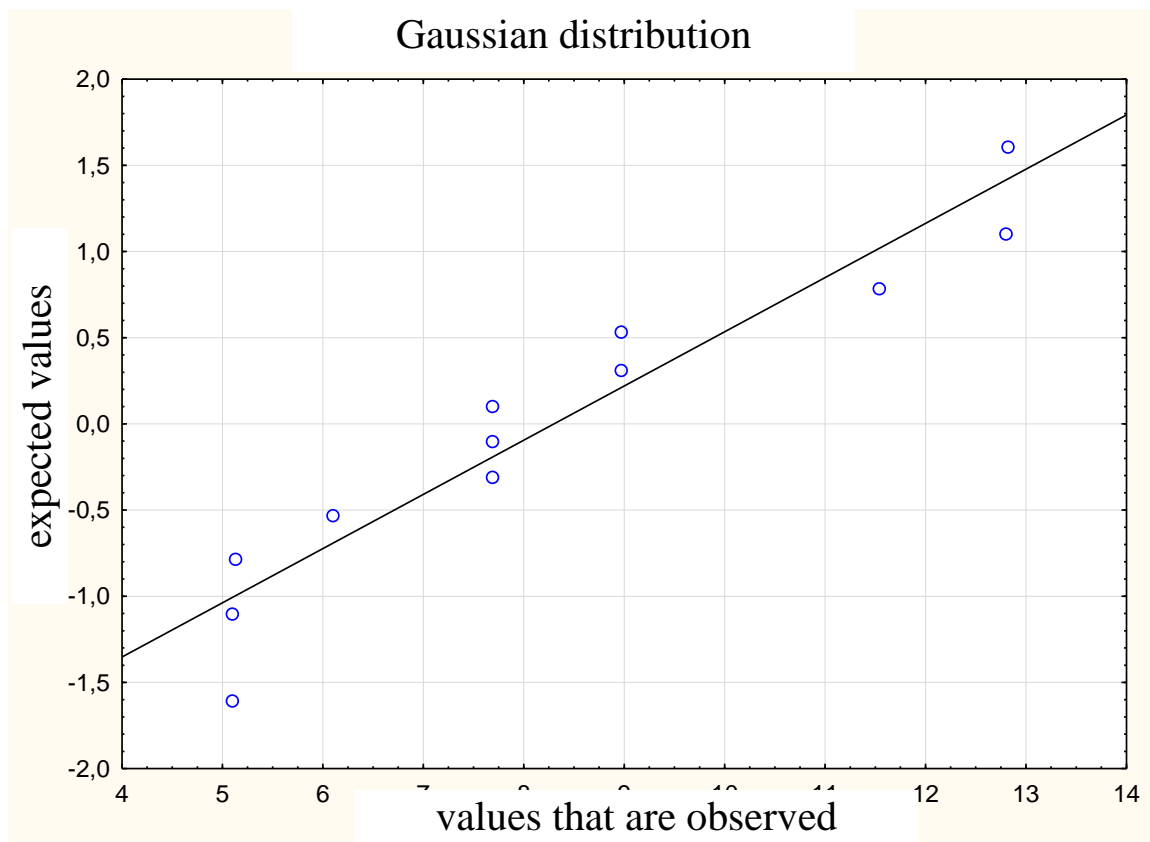

Fig. 1. Graph for checking the normal distribution of the number of patients (confidence probability 0.1 )

According to our observations, the share of visits to the outpatient urologist for prostatitis is from $46.2 \%$ to $58.0 \%$ per month of the total, and this is a fairly steady trend that repeats from month to month and year to year, characterized by high values correlation coefficient ranging from 0.78 to 0.94 (average correlation coefficient for the entire study period 0.85). The distribution of the total number of prostatitis patients during the year, expressed as a percentage, is presented in Table 3.

Table 3

Distribution in percent by months of prostatitis patients who sought urologist aid during $2011-2013$

\begin{tabular}{|l|c|c|c|c|c|c|c|c|c|c|c|c|}
\hline $\begin{array}{l}\text { month } \\
\text { year }\end{array}$ & 1 & 2 & 3 & 4 & 5 & 6 & 7 & 8 & 9 & 10 & 11 & 12 \\
\hline 2011 & 6,9 & 5,5 & 5,5 & 8,3 & 11,2 & 12,5 & 12,8 & 8,3 & 8,3 & 6,9 & 8,3 & 5,5 \\
\hline 2012 & 5,1 & 6,1 & 5,1 & 7,7 & 9,1 & 11,5 & 12,8 & 12,8 & 9,2 & 7,8 & 7,7 & 5,1 \\
\hline 2013 & 5,5 & 5,3 & 5,8 & 7,9 & 11,9 & 12,1 & 12,8 & 9,1 & 8,4 & 8,2 & 7,4 & 5,6 \\
\hline
\end{tabular}


The table shows that these indicators are unevenly distributed throughout the year. Thus, the largest number of appeals occurs in the summer months $(\mathrm{p}<0.05)$. Visualization of the result of the process of identification of the data presented in Table. 3, is shown in Fig. 2. On the abscissa axis are time intervals of observation (in months), and on the ordinate axis the percentage of visits to the urologist).

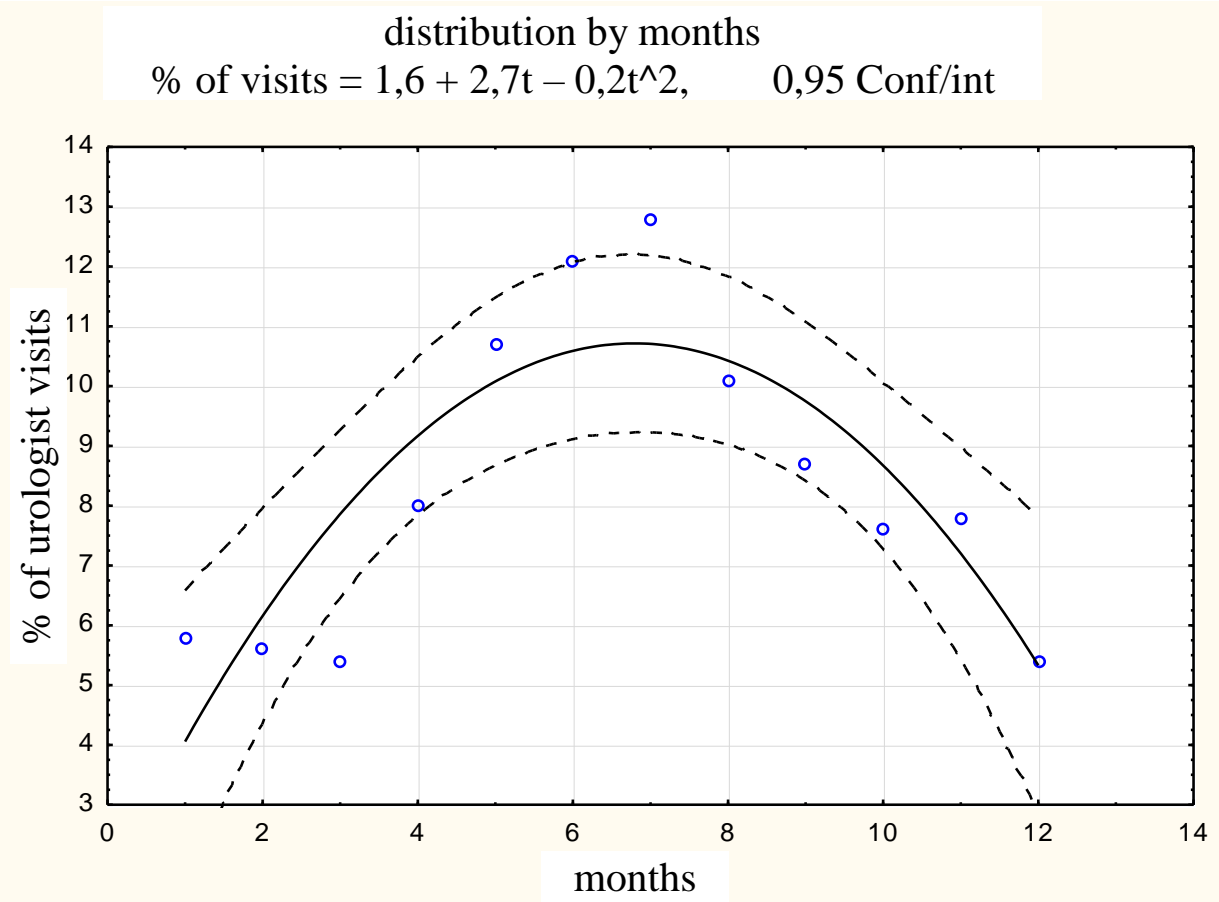

Fig. 2. Dependence of the percentage of visits to urologist by prostatitis patients on the month in the annual calculation (confidence interval 0.95)

We approximated by the statistical model in the form of a second-degree polynomial (percentage of visits to the doctor in percentage terms) the patients' visits to the urologist during the year with a confidence level of 0.95 . Attendance rate $(\%)=1.6+2.7 \times \mathrm{t}-0.2 \times \mathrm{t} 2$ (1), where $t$ is the number of the month in the year.

In order to build a predictive model, we conducted a structural and parametric identification of the model of visits to the urologist. According to the first three years included in the study, we built an approximate model (with a confidence interval of 0.95 ) of visits to the urologist (according to formula 1), and in 2017 data we are testing it. The coefficients of the model were obtained in the demo - version of Statistica-12 package by the standard method of least squares in the sample 2011 - 2015.

Preliminary statistical analysis showed that the obtained model is adequate, since the real data are within the confidence interval of the obtained model. This indicates that the 
general trend of patients with prostatitis seeking urologist's aid does not change significantly from year to year. For comparison, the projected values for 2017 and the actual data are presented in Table 4.

Table 4

Comparison of calculated and real indicators of patients' visits in 2017, percentage

\begin{tabular}{|c|c|c|c|c|c|c|c|c|c|c|c|c|}
\hline month & 1 & 2 & 3 & 4 & 5 & 6 & 7 & 8 & 9 & 10 & 11 & 12 \\
\hline $\begin{array}{c}\text { indicator } \\
\begin{array}{c}\text { Predicted } \\
\text { values }\end{array}\end{array}$ & 4,1 & 6,2 & 7,9 & 9,2 & 10,1 & 10,6 & 10,7 & 10,4 & 9,7 & 7,6 & 7,1 & 5,2 \\
\hline Real data & 5,4 & 5,3 & 5,9 & 7,4 & 11,2 & 12,9 & 13,3 & 9,2 & 8,1 & 7,6 & 7,2 & 6,5 \\
\hline
\end{tabular}

The studied model was obtained on the basis of structural and parametric identification of the model of changes in the number of diseases depending on the calendar month. It was tested on a control sample (on the incidence of 28 patients) according to Wilk's test and proved its adequacy. The maximum forecast error was $12 \%$.

As a result, the obtained model, assuming the validity of K. Shannon's hypothesis, can be used for preliminary prediction when planning the work of the urological consulting room for this type of disease, for any period of time, as the general trend of chronic prostatitis morbidity during the year.

As seen from Fig. 2, the dependence of the number of diseases is fluctuating in nature, with a pronounced maximum in the summer. This fact confirms the validity of the hypothesis about the presence of periodicity and seasonality for this type of urological diseases.

Since it was found that there is a certain pattern in the patients' seek for medical care during the year, further, in order to find the period in the study data set, we, taking into account the normality test, applied the spectral method (Fourier analysis) and obtained the periodogram presented in Fig. 3.

In essence, this graph is an integral generalization of the whole set of data obtained over three years, so to speak, "convolution". Due to the fact that it is not stretched for the entire period of the study, but "folded" thrice, we can clearly distinguish the main patterns of the process. The shift of the maxima of the periods occurs within the "noise" of the measurements.

The type of changes in Fig. 3 indicates the repetitive nature of the process by months (in the annual comparison period). Analyzing the obtained dependence on the time axis (abscissa), it should be noted two characteristic, pronounced peaks, corresponding to 6 and 12-month periods. The peak, which corresponds to a period of 12 months, is of the greatest 
importance. The presence of the main maximum, corresponding to a period of 12 months, indicates that the data obtained from the processing of patients' appeals have an annual frequency and a pronounced seasonal component, and the time series itself is non-stationary.

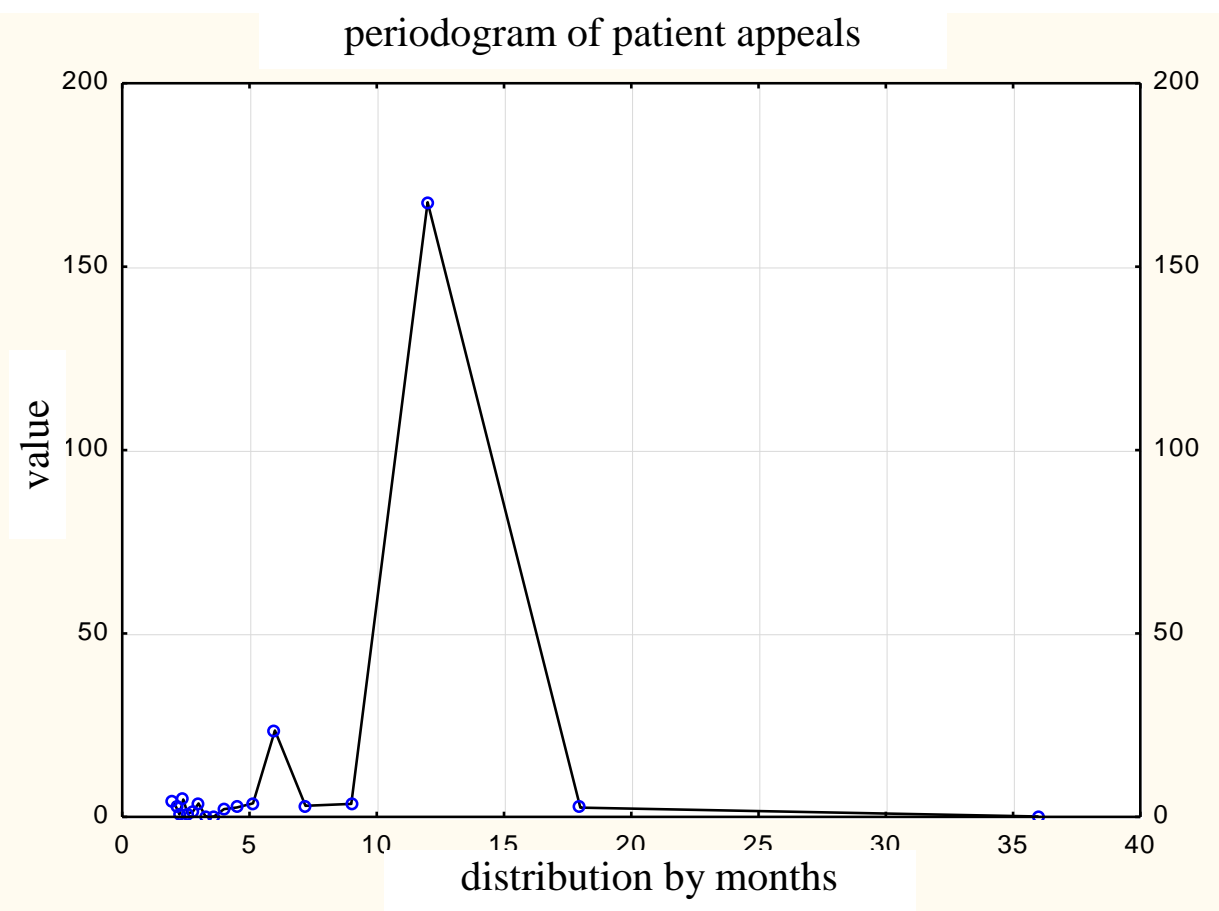

Fig. 3 Periodogram of patients' visits to the urologist (in months)

Conclusions. 1. The largest proportion of viral etiology prostatitis patients who sought medical aid is between $20-29$ y.o. The average duration of the disease increases with age.

2. Verification of the normality of the distribution proved that the methods of mathematical statistics can be applied to the presented sample. Analysis and visualization of the data obtained shows that the incidence increases from May to August, decreases in the winter months and early autumn.

3. Patients' visits to the urologist were approximated by a statistical model in the form of a second-degree polynomial. The results of testing the obtained model proved its effectiveness and feasibility as a prognostic. The extrapolation functional - a polynomial of degree 2 , selected under the conditions of parametric statistics as a predictive model, provided a fairly good approximation of the model to the empirical data in the selected confidence interval with a significance level of 0.1 .

4. In order to confirm the seasonality of fluctuations in medical care for patients with prostatitis using a spectral method, a periodogram was constructed, which confirmed the presence of annual periodicity. 
5. This trend is repeated annually with a high correlation (0.85). The shift of the maxima of the periods occurs within the "noise" of the measurements.

\section{References:}

1. The 2013 EAU Guidelines on Chronic Pelvic Pain: Is Management of Chronic Pelvic Pain a Habit, a Philosophy, or a Science? 10 Years of Development / D. S. Engeler, A. P. Baranowski, P. Dinis-Oliveira [et al.] // Eur. Urol. — 2013. — Vol. 64 (3). - P. 431 439.

2. Nagorny A.E. Pathomorphosis of clinical manifestations in genital herpes, chlamydia and trichomoniasis / A.E. Nagorny // Dermatology and venereology. - 2011. - № 3 (53). - p. 34 - 43.CDC. Sexually transmitted diseases treatment guidelines. MMWR Recomm Rep. - 2015. — № 64. - P. 1 - 137.

3. CDC. Sexually transmitted diseases treatment guidelines. MMWR Recomm Rep. - 2015. - № 64. - P. 1 - 137.

4. Sexually Transmitted Infections: A Medical Update / P. R. Jennings, R. W. Flenner // Physician Assistant Clinics. - 2017. — Vol. 2, Issue 2. - P. 207 - 218.

5. Smirnov V. A. Drug therapy of chronic prostatitis / V. A. Smirnov // PHARMIndex-Praktik. - 2006. - № 10. - p. 46 - 55.

6. Kazmirchuk V.E. Clinic, diagnosis and treatment of human herpes virus infections: monograph / V.E. Kazmirchuk, D.V. Maltsev / National Medical University. A. A. Bogomolets; Institute of Immunology and Allergology. - К .: Phoenix, 2009. - 248 р.Дуда О. K.

7. Herpetic and herpesvirus infection. Training Manual for Doctors / O. K. Dud, M. I. Krasnov, V. M. Kozko. - Kyiv: NMAPE, 2015. - 96 p.

8. On the issue of herpetic infection as an actual problem of the present / VPP Borak, L. B. Romanyuk, V.T. Borak [and others] // Actual infectology. - 2016 - No. 2 (11). P. 53 - 58.

9. Herpes virus infections (clinic, diagnosis and therapy): proc. allowance / Small V.P., Polukchi A.K., Shvaychenko A.A. [and others] / Kharkiv Medical Academy of Postgraduate Education [V. P. Maliy (commonly ed.), A.K. Polukchi (obshch. Ed.)]. - H .: Prapor, 2008. - 208 p.

10. Mavrov GI. Influence of viruses of the herpes group on the development of the pathology of the nervous system in dermatological patients: a review of the literature and our 
own observations / GI Mavrov, ME Zapolsky // International Neurological Journal. - 2013. № 5 (59). - access mode: http: //www.mifua.com/archive/article/36755.

11. Prostatitis / [Ed. P. A. Shcheleva]. - M .: MEDpress-inform, 2007. - 224 p

12. Primary, general morbidity, mortality from major diseases of the organs of the genitourinary system in the aspect of the activity of the DU "Institute of Urology of NAMS of Ukraine" / S.O. Vozianov, N.O.Saydakov, V.M. Grigorenko [and others] // Urology . - 2015. T. 19, No. 3. - P. 15 - 28.

13. Gorilovskiy L.M. Chronic prostatitis / L.M. Gorilovskiy, M. B. Zingerenko // Leading doctor. - 2003. - № 7. - p. 4 - 8.

14. Wagenlehner F. National Institutes of Health Chronic Prostatitis Symptom Index (NIH-CPSI) Symptom Evaluation in Multinational Cohorts of Patients with Chronic Prostatitis / Chronic Pelvic Pain Syndrome / F. Wagenlehner, O. van Tillb, V. Magric [et al.] // Eur. Urol. - 2013. - V. 63 (5). - P. 953 - 959.

15. Borovikov V.P. STATISTICA. Art analysis danih on computers: for professionals. 2nd view SD. - SPb .: Peter, 2003. - 688 p.

16. Lapach S. N. Statistical methods in biomedical research using Excel / S. N. Lapach, A. V. Chubenko, P. N. Babich. - K .: MORION, 2000. - 320 p.

17. Gerasyevich VA Computer for a doctor: Samovichitel. Kind. 2nd, correct And add / V.A. Herasevich. - SPb .: BHV-Petersburg, 2004. - 488 p.

17. Topolnik VG Mathematical-statistical methods of research [taught. manual for studio higher tutor Z.] / V.G. Topolnik / Donetsk National University of Economics and Trade them. Michael Tugan-Baranovsky. Department of organization and quality management of restaurant economy. -Donetsk: DonNUET, 2009. - 176 p.

18. Novikov MV Estimation of the seasonal morbidity of herpesvirus infection of the urogenital sphere in Odessa / MV Novikov, F. I. Kostev, V. S. Goydyk // Bulletin of Marine Medicine. - 2016 - No. 1 (70). - P. 27 - 33. 